\title{
Pré-escolar com Síndrome de Megalencefalia - má formação capilar (MCAP): relato de caso
}

\author{
Pre-school with Megalencephaly Syndrome - capillary \\ malformation (MCAP): case report
}

\section{Preescolar con Síndrome de Megalencefalia - malformación capilar (MCAP): reporte de um caso}

\author{
Gustavo Bittencourt Camilo슬 Gabriela Cumani Toledo Camilo², Vitória \\ Dias Campos ${ }^{3}$, Pedro de Freitas Batista Mendes ${ }^{4}$, Matheus Cruz \\ Ferraro ${ }^{5}$, Lara Esteves Ferreira de Oliveira Almeida ${ }^{6}$, Fernando \\ Oliveira Nunes Caixeta7, Cristiano Guimarães Pinto Martins ${ }^{5}$
}

\begin{abstract}
1.Departamento de Radiologia do Hospital e Maternidade Terezinha de Jesus (HMTJ) e professor da Faculdade de Ciências Médicas e da Saúde de Juiz de Fora (SUPREMA). Programa de Pós Graduação em Saúde Brasileira da Universidade Federal de Juiz de Fora. Juiz de ForaMG, Brasil. http://orcid.org/0000-0001-7387-8381

2.Departamento de Radiologia do Hospital e Maternidade Terezinha de Jesus (HMTJ) e professor da Faculdade de Ciências Médicas e da Saúde de Juiz de Fora (SUPREMA). Programa de Pós Graduação em Saúde Brasileira da Universidade Federal de Juiz de Fora. Juiz de ForaMG, Brasil. http://orcid.org/0000-0003-2654-8894

3.Estudante de graduação da Faculdade de Ciências Médicas e da Saúde de Juiz de Fora (FCMS JF/SUPREMA). Juiz de Fora-MG, Brasil. https://orcid.org/0000-0001-8538-0046

4.Estudante de graduação de Faculdade de Medicina da Universidade Federal de Juiz de Fora (UFJF). Juiz de Fora-MG, Brasil. https://orcid.org/0000-0001-6468-3515

5.Estudante de graduação de Faculdade de Medicina da Universidade Federal de Juiz de Fora (UFJF). Juiz de Fora-MG, Brasil. http://orcid.org/0000-0002-9900-8453

6. Estudante de graduação da Faculdade de Ciências Médicas e da Saúde de Juiz de Fora (FCMS JF/SUPREMA). Juiz de Fora-MG, Brasil. https://orcid.org/0000-0002-4497-8065

7.Estudante de graduação da Faculdade de Ciências Médicas e da Saúde de Juiz de Fora (FCMS JF/SUPREMA). Juiz de Fora-MG, Brasil. https://orcid.org/0000-0002-2397-0427

8.Faculdade de Educação Física da Universidade Federal de Juiz de Fora. Juiz de Fora-MG, Brasil. https://orcid.org/0000-0003-4832-4824
\end{abstract}

\section{Resumo}

Relato de caso da síndrome de Megalencefalia - Má formação Capilar para divulgar informações e propiciar a discussão sobre síndromes congênitas. Nesse contexto, são recomendados acompanhamentos clínico e radiológico para monitorar a evolução da síndrome e, quanto à abordagem terapêutica, esta deve ser a mais ampla e multidisciplinar possível, para determinar evoluções neurais, psicomotoras e na adaptação do indivíduo ao ambiente uma vez que um tratamento específico inexiste.

Unitermos. Megalencefalia; Malformação de Arnold-Chiari; Instabilidade Articular; Hemangioma Capilar

\footnotetext{
Abstract

Case report of Megalencephaly syndrome - Capillary malformation to disseminate information and encourage discussion about congenital syndromes. In this context, clinical and radiological follow-ups are recommended to monitor the evolution of the syndrome and, as for the therapeutic approach, it should be as broad and multidisciplinary as possible, to determine
} 
neural, psychomotor therapy and adaptation of the individual to the environment once a treatment does not exist.

Keywords. Megalencephaly; Arnold-Chiari Malformation; Joint Instability; Hemangioma, Capillary

\section{Resumen}

Reporte de caso de síndrome de Megalencefalia - Malformación capilar para difundir información y fomentar la discusión sobre síndromes congénitos. En este contexto, se recomiendan los seguimientos clínicos y radiológicos para monitorizar la evolución del síndrome $y$, en cuanto al abordaje terapéutico, debe ser lo más amplio y multidisciplinar posible, para determinar neural, psicomotoras y adaptación del individuo al entorno, ya que no existe un tratamiento específico.

Palabras clave: Megalencefalia; Malformación de Arnold-Chiari; Inestabilidad conjunta; Hemangioma capilar

Trabalho realizado no Departamento de Radiologia da Faculdade das Ciências Médicas e da Saúde de Juiz de Fora - SUPREMA, Juiz de Fora-MG, Brasil.

\section{INTRODUÇÃO}

A Síndrome de Megalencefalia - Malformação Capilar (megalencephaly-capillary malformations syndrome MCAP) foi primeiramente descrita em 1997 como Macrocefalia-Cutis Marmorata Telangiectatica congênita (M(MTC) $)^{1,2}$ e, desde então, há cerca de 150 relatos na literatura ${ }^{3}$. Dentre os reportados, estabeleceu-se igualdade de prevalência entre gêneros e etnias ${ }^{4}$.

Trata-se de uma rara síndrome genética fenotipicamente caracterizada pelo envolvimento do Sistema Nervoso Central e de anomalias vasculares, com destaque principal a megalencefalia congênita ou pós-natal, ventriculomegalia e anormalidades cerebrais corticais, máculas cor-de-rosa ou avermelhadas na pele, sindactilia e 
polidactilia, acrescido de um supercrescimento focal ou segmentar de estruturas físicas ${ }^{3-11}$.

Atualmente o reconhecimento da síndrome é realizado clinicamente, apesar da diversidade de critérios diagnósticos entre os autores ${ }^{7,8}$. Todavia, há achados radiológicos e anatômicos frequentes entre os pacientes, como ventriculomegalia acompanhada ou não de hidrocefalia, assimetria e polimicrogiria da malformação cortical, cerebelo e fossa posterior aumentados, ectopia amigdaliana cerebelar e corpo caloso espessado ${ }^{2-8}$.

Recentemente, descobriu-se uma associação entre a síndrome MCAP e mutações no gene PIK3CA ${ }^{10}$. Entretanto, ainda há uma disparidade entre o conhecimento científico acerca dos sinais e sintomas característicos e da história natural da doença, sendo necessária, portanto, ampla abordagem terapêutica multidisciplinar, uma vez que um tratamento específico ainda inexiste ${ }^{4,9}$. Dessa maneira, almeja-se que o relato seja uma forma de divulgar informações e propiciar o debate acerca desse grupo de síndromes congênitas.

\section{MÉTODO}

O presente estudo foi enviado ao comitê de ética com aprovação sob o número CAAE: 11320919.1.0000.5103. Os exames de imagem fazem parte do arquivo pessoal dos membros da equipe e todas as imagens tiveram sua veiculação aprovada pelo responsável legal. 
Criança de 4 anos, feminino, em acompanhamento multidisciplinar atual com fisioterapeuta, fonoaudiólogo, terapeuta ocupacional e neurologista. Houve diagnóstico ultrassonográfico de polidramnia e macrocefalia no exame pré-natal. Após o nascimento, realizou-se investigação genética, exame físico minucioso e múltiplos exames de imagem que evidenciaram hipermobilidade de múltiplas articulações, pequenas máculas cutâneas avermelhadas, protuberância frontal, atraso no desenvolvimento motor e da fala, macrocefalia, hidrocefalia, polimicrogiria, assimetria dos hemisférios cerebrais, alterações na intensidade do sinal cerebral, aumento do volume da fossa posterior, ectopia das amígdalas (os dois últimos achados relatados na Síndrome de Chiari) e cisto do septo pelúcido, conforme apresentado nas Figuras 1 e 2. Assim, a associação dos achados nos exames de imagem com a macrocefalia, bem como os achados cutâneos propiciaram o diagnóstico da Síndrome de MCAP.

A alteração no septo pelúcido ocasionou compressões do terceiro ventrículo e do aqueduto cerebral, que, por sua vez, acarretaram o agravamento da hidrocefalia e da hipertensão craniana, sendo necessário a realização de três descompressões cirúrgicas até dois anos de idade. Após a última cirurgia, a criança apresentou estabilidade do quadro, com melhora do bem estar geral e evolução do desenvolvimento motor, possibilitando-a engatinhar, sentarse e alimentar-se. 

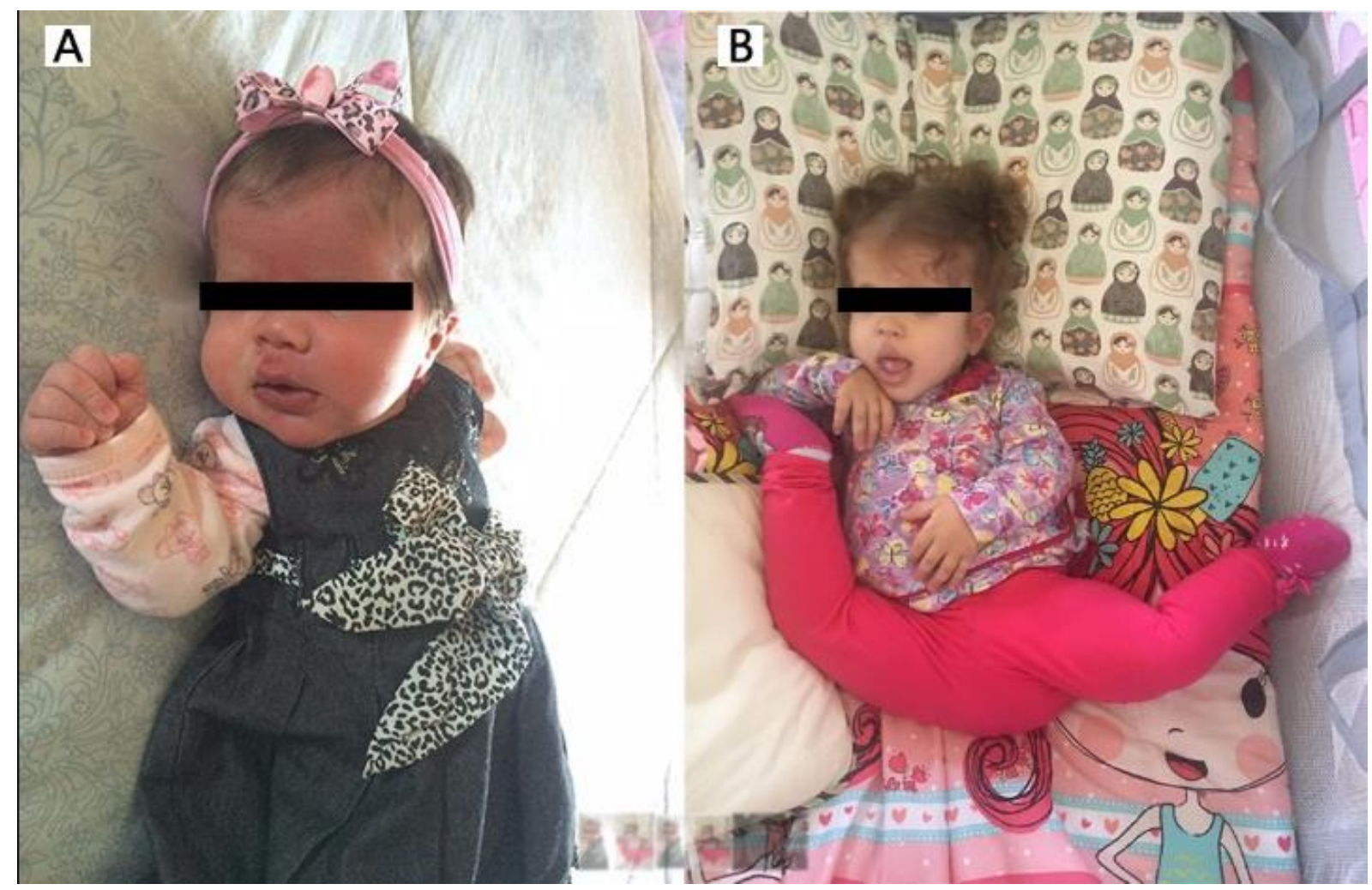

Figura 1. Imagens A e B demonstram máculas cutâneas avermelhadas, protuberância frontal, aumento $\mathrm{da}$ espessura cutânea, macrocefalia e hipermobilidade articular.

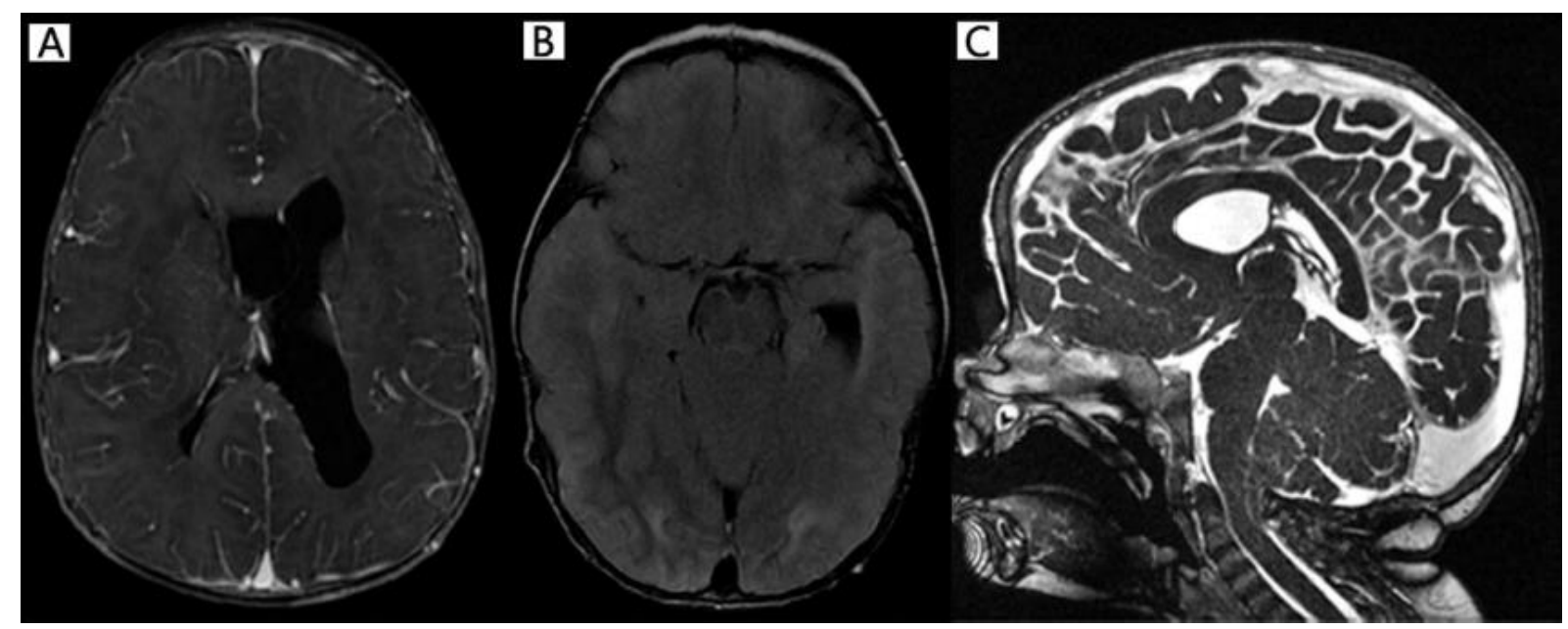

Figura 2. Ressonância magnética cerebral mostrando em A corte axial pós contraste ponderada em T1 demonstrando o cisto do septo pelúcido e hidrocefalia. Em B axial T1 sem contraste ressaltando heterotopia associado à alteração na intensidade da substância branca na região occipital bilateral. Em C corte sagital na ponderação T2, demonstrando malformação de Chiari, cisto de septo pelúcido, aumento de volume da fossa posterior e ectopia de amígdalas. 
O acompanhamento foi realizado de forma multidisciplinar não homogênea e com profissionais liberais, sem a participação completa constante de nenhuma instituição hospitalar, embora ocorra desde o nascimento até o presente dia. A paciente realiza seguimento com neurologista pediátrico cerca de 2 a 3 vezes ao ano e com orientações sempre que forem necessárias. O acompanhamento fisioterápico ocorre desde a idade de 1 ano, com frequência de 2 vezes por semana, com inspiração no instrumento "Teste de Triagem de Desenvolvimento Denver $\mathrm{II}^{\prime \prime 2}$. Em relação à fonoaudiologia, o acompanhamento é realizado desde os 2 anos de idade, semanalmente, inspirado no "Protocolo de observação de comportamentos"13. Finalmente, a criança realiza natação e acompanhamento com terapeuta ocupacional de maneira semanal, com orientação individualizada, porém sem nenhum modelo específico de sistematização protocolar. Nesse contexto, denota-se melhoras significativas, como maior mobilidade, desenvolvimento da postura e do tônus muscular, além de ser capaz de manter-se de muleta.

\section{DISCUSSÃO}

As síndromes de supercrescimento (overgrowth syndromes - OS) compreendem um grupo heterogêneo de condições caracterizadas pela proliferação excessiva de diversos tecidos, levando ao crescimento generalizado ou segmentar e simétrico ou assimétrico dos mesmos ${ }^{10,14,15}$. 
Esses distúrbios podem se manifestar ao nascimento ou desenvolver-se na primeira infância3,10,11,14. Existe uma grande variedade de OS citadas pela literatura, dentre elas: MCAP (megalencephaly-capillary malformations syndrome), DMEG (dysplastic megalencephaly), CLOVES (Congenital Lipomatous Overgrowth, Vascular Malformations, Epidermal Nevi, Scoliosis/Skeletal and Spinal syndrome), HHML (hemihyperplasia-multiple lipomatosis), Fibroadipose hyperplasia or Overgrowth (FAO) $)^{7,10,14,16}$. Na maioria dos casos, esses distúrbios são causados por mutações somáticas em mosaico nos genes associados à via celular $\mathrm{PI3K} / \mathrm{AKT} / \mathrm{mTOR}$, de forma que uma disfunção nessa via pode promover um rápido aumento na expressão do receptor de insulina, sendo essa provavelmente a base fisiopatológica de algumas das síndromes de crescimento excessivo ${ }^{11,14}$.

Dentre as OS, destaca-se a síndrome de megalencefalia, descrita pela primeira vez em 19973,8,15. A MCAP está relacionada a mutações no gene PIK3CA importante para o crescimento e metabolismo da maioria das células ${ }^{15}$. Ademais, também foram descritos casos familiares da síndrome, sugerindo herança autossômica recessiva ou mosaicismo da linha germinativa ${ }^{8,15}$. Tal distúrbio é fenotipicamente caracterizado por anomalias vasculares e pelo envolvimento do Sistema Nervoso Central (SNC), marcado por megalencefalia congênita ou pós-natal, ventriculomegalia (que pode levar a ectopia tonsilar cerebelar progressiva levando a malformação de Chiari) e 
polimicrogiria $3,5,10,16$.

Consequentemente, são evidenciados sintomas neurológicos secundários, como atraso no desenvolvimento, hipotonia, convulsões, características autistas e problemas comportamentais, como irritabilidade inexplicável, déficit de atenção, distúrbio de hiperatividade e transtorno obsessivocompulsivo $3,4,10$.

Em relação às anomalias vasculares, encontra-se especialmente malformações capilares da face e da linha média do corpo - que formam máculas cor-de-rosa ou avermelhadas na pele, anomalias distais dos membros, sindactilia e polidactilia, somado a um supercrescimento focal ou segmentar de estruturas físicas ${ }^{3,5,16}$. Ao nascimento também é tipicamente notado certo grau de macrocefalia, decorrente da megalencefalia, o que, em muitos casos, torna a MCAP um distúrbio clinicamente reconhecível ${ }^{10,16}$.

Tendo em vista a variedade de apresentações e sintomas, muitas vezes são necessárias inúmeras investigações para a confirmação dessa condição genética ${ }^{8,10}$. Neste contexto, não há um consenso acerca dos critérios a serem utilizados ${ }^{4,6,7}$. O diagnóstico é feito com base apenas em critérios clínicos e, dentre os modelos existentes, são três os autores referência que se destacam $4,6,7,16$.

Robertson et al. sugeriram que o diagnóstico pode ser feito em um paciente que tem cutis marmorata telangiectatica congenital (CMTC) e macrocefalia congênita, 
juntamente com pelo menos quatro das seguintes características: hipotonia neonatal, atraso no desenvolvimento, defeito do tecido conjuntivo da pele ou articulações, protuberância frontal, malformações capilares na linha média da face, sindactilia cutânea dos dedos dos pés, supercrescimento assimétrico parcial e hidrocefalia 6 .

Franceschini et al sugeriram a macrocefalia como principal característica e pelo menos dois dos seguintes achados: supercrescimento, cutis marmorata, angiomata, polidactilia/sindactilia e assimetria ${ }^{6}$.

Wright et al sugeriram que o diagnóstico requer a presença de macrocefalia e malformação capilar, associados à pelo menos dois dos seguintes critérios menores: assimetria ou supercrescimento, atraso no desenvolvimento, malformações capilares faciais ou da linha média, hipotonia neonatal, sindactilia/polidactilia, protuberância frontal, hipermobilidade articular ou hiperelasticidade da pele e hidrocefalia6.

Quanto aos exames de imagem da MCAP, achados tipicamente visualizados são ventriculomegalia (VMEG) que pode progredir para hidrocefalia (HYD), assimetria especialmente dos ventrículos laterais, polimicrogiria com malformação cortical (PMG), cerebelo aumentado - levando ao apinhamento da fossa posterior, ectopia amigdaliana cerebelar (CBTE ou herniação) e corpo caloso espessado (mega corpus callosum) 2-8,15.

São recomendados acompanhamentos clínico e radiológico para monitorar a evolução progressiva da 
síndrome, com frequentes ressonâncias magnéticas (RM), com especial atenção para a avaliação da fossa posterior, tendo em vista o alto risco de HYD e CBTE ${ }^{3,6}$. Também é indicada a realização de ultrassonografia abdominal a cada 3 a 4 meses até os 8 anos de idade somada à monitorização neurológica e RM da coluna vertebral em pacientes com envolvimento troncular, a fim de excluir a presença de neurofibromas nervosos ou lesões vasculares/lipomatosas $3,4,6$.

No que se refere à abordagem terapêutica, esta deve ser a mais ampla e multidisciplinar possível, uma vez que um tratamento específico ainda é inexistente ${ }^{4,8,9}$. Segue-se, portanto, o tratamento sintomático, enquanto a história natural desse distúrbio ainda visa ser esclarecida7,8,10. $\mathrm{Na}$ presença dos sinais e sintomas clínicos, é necessária a avaliação genética em um estágio inicial, além da submissão a uma análise molecular - especialmente em casos em que geram dúvidas - a fim de alcançar o diagnóstico correto e estabelecer o seguimento e tratamento mais adequados ${ }^{10,16}$.

\section{CONCLUSÃO}

Apesar de ser uma síndrome de múltiplas características definidas, a escassa prevalência da síndrome de MCAP acarreta a ausência de consenso dos métodos diagnósticos estabelecidos, fato que, em alguns casos, dificulta sua identificação e sua abordagem terapêutica, a qual deve ser a mais ampla e multidisciplinar possível, de 
forma a determinar evoluções neurais e psicomotoras, uma vez que um tratamento específico ainda inexiste.

\section{REFERÊNCIAS}

1.Clayton-Smith J, Kerr B, Brunner $H$, Tranebjaerg L, Magee A, Hennekam RC, et al. Macrocephaly with cutis marmorata, haemangioma and syndactyly - a distinctive overgrowth syndrome. Clin Dysmorphol 1997;6:291-302.

http://dx.doi.org/10.1097/00019605-199710000-00001

2. Moore CA, Toriello HV, Abuelo DN, Bull MJ, Curry CJ, Hall BD, et al. Macrocephaly-cutis marmorata telangiectatica congenita: A distinct disorder with developmental delay and connective tissue abnormalities. Am J Med Genet 1997;70:6773.

http://dx.doi.org/10.1002/(sici)10968628(19970502)70:1<67: :aidajmg13>3.0.co;2-v

3. Mirzaa GM, Conway RL, Gripp KW, Lerman-Sagie T, Siegel DH, deVries LS, et al. Megalencephaly-capillary malformation (MCAP) and megalencephaly-polydactyly-polymicrogyria-hydrocephalus (MPPH) syndromes: two closely related disorders of brain overgrowth and abnormal brain and body morphogenesis. Am J Med Genet Part A 2012;158A:269-91. http://dx.doi.org/10.1002/ajmg.a.34402

4.Loughan AR, Harrell M, Perna R, Allen A, Suddarth B. Megalencephaly-capillary Malformation Polymicrogyria: A Review and Complex Pediatric Case Report. Appl Neuropsychol Child 2017;6:36977. http://dx.doi.org/10.1080/21622965.2016.1182432

5. Mirzaa GM, Rivière JB, Dobyns WB. Megalencephaly syndromes and activating mutations in the PI3K-AKT pathway: MPPH and MCAP. Am J Med Genet Part C 2013;163C:122-30.

http://dx.doi.org/10.1002/ajmg.c.31361

6. Papetti L, Tarani L, Nicita F, Ruggieri M, Mattiucci C, Mancini F, et al. Macrocephaly-capillary malformation syndrome: description of a case and review of clinical diagnostic criteria. Brain Dev 2012;34:143-7. http://dx.doi.org/10.1016/j.braindev.2011.02.001

7.Segal D, Heary RF, Sabharwal S, Barry MT, Ming X. Severe holocord syrinx in a child with megalencephaly-capillary malformation syndrome. Neurosurg Pediatr 2016;18:79-82.

http://dx.doi.org/10.3171/2016.1.peds15482

8. Alsaedi SA, Qurashi O, Bajunaid M, Altalhi AA, Shawli AM. One of the First Cases with PIK3CA-related Overgrowth Spectrum (PROS) in Saudi Arabia: A Case Report and Literature Review. Cureus 2020;12: e6586. http://dx.doi.org/10.7759/cureus.6586

9.Conway RL, Pressman BD, Dobyns WB, Danielpour M, Lee J, Sanchez-Lara PA, et al. Neuroimaging findings in capillary malformation: a longitudinal study of 17 patients. Am J Med Genet A 2007;143A:2981-3008. http://dx.doi.org/10.1002/ajmg.a.32040 
10. Maguolo A, Antoniazzi F, Span A, Fiorini E, Gaudino R, Mauro M, et al. Clinical pitfalls in the diagnosis of segmental overgrowth syndromes: a child with the c.2740G > a mutation in PIK3CA gene. Ita J Ped 2018;44:2-6. http://dx.doi.org/10.1186/s13052-018-0568-8

11.Davis S, Ware MA, Zeiger J, Deardorff MA, Grand K, Grimberg A, et al. Growth hormone deficiency in megalencephaly-capillary malformation syndrome: An association with activating mutations in PIK3CA. Am J Med Genet Part A 2020;182:162-8. http://dx.doi.org/10.1002/ajmg.a.61403

12. Drachler $M$ de $L$, Marshall T, de Carvalho Leite JC. A continuousscale measure of child development for population-based epidemiological surveys: a preliminary study using Item Response Theory for the Denver Test. Paed Perin Epidemiol 2007;21:13853. http://dx.doi.org/10.1111/j.1365-3016.2007.00787.x

13.Chiari BM, Basilio CS, Nakagawa EA, Cormedi MA, Silva NSM, Cardoso RM, et al. Proposta de sistematização de dados da avaliação fonoaudiológica através da observação de comportamentos de criança de 0 a 6 anos. Pró-fono 1991;3:29-36.

14.Lacerda LS, Alves UD, Zanier JFC, Machado DC, Camilo GB, Lopes AJ. Differential Diagnoses of Overgrowth Syndromes: The Most Important Clinical and Radiological Disease Manifestations. Radiol Res Pract 2014;2014:947451. http://dx.doi.org/10.1155/2014/947451

15.Choi YC, Yum MS, Kim MJ, Lee YJ, Ko TS. Megalencephaly-capillary malformation-polymicrogyria syndrome: the first case report in Korea. Korean J Pediatr 2016;59:152-6. http://dx.doi.org/10.3345/kjp.2016.59.11.s152

16.McDermott JH, Byers $\mathrm{H}$, Clayton-Smith J. Detection of a mosaic PIK3CA mutation in dental DNA from a child with megalencephaly capillary malformation syndrome. Clin Dysmorphol 2015;25:16-8. doi.org/10.1097/MCD.0000000000000099 